\title{
Role of $N^{*}(1535)$ in the $\Lambda_{c}^{+} \rightarrow \bar{K}^{0} \eta p$ decay and the possible $\phi p$ state in the $\Lambda_{c}^{+} \rightarrow \pi^{0} \phi p$ decay
}

\author{
Ju-Jun Xie ${ }^{1,2,3, *}$, Li-Sheng Geng ${ }^{4,3}$, and Feng-Kun Guo ${ }^{5,2}$ \\ ${ }^{1}$ Institute of Modern Physics, Chinese Academy of Sciences, Lanzhou 730000, China \\ ${ }^{2}$ School of Nuclear Science and Technology, University of Chinese Academy of Sciences, Beijing 100049, China \\ ${ }^{3}$ School of Physics and Microelectronics, Zhengzhou University, Zhengzhou, Henan 450001, China \\ ${ }^{4}$ School of Physics \& Beijing Advanced Innovation Center for Big Data-based Precision Medicine, Beihang University, Beijing 100191, \\ China \\ ${ }^{5}$ CAS Key Laboratory of Theoretical Physics, Institute of Theoretical Physics, Chinese Academy of Sciences, Zhong Guan Cun East \\ Street 55, Beijing 100190, China
}

\begin{abstract}
The nonleptonic weak decays of $\Lambda_{c}^{+} \rightarrow \bar{K}^{0} \eta p$ and $\Lambda_{c}^{+} \rightarrow \pi^{0} \phi p$ are investigated from the viewpoint of probing the $N^{*}(1535)$ resonance and the possible $\phi p$ state. For the $\Lambda_{c}^{+} \rightarrow \bar{K}^{0} \eta p$ decay, we study the invariant mass distribution of $\eta p$ with both the chiral unitary approach and an effective Lagrangian model. Within the chiral unitary approach, the $N^{*}(1535)$ resonance is dynamically generated from the final state interaction of mesons and baryons in coupled channels. While for the effective Lagrangian model, we take a Breit-Wigner formula for the $N^{*}(1535)$ resonance. We found that the behavior of the $N^{*}(1535)$ resonance in the $\Lambda_{c}^{+} \rightarrow$ $\bar{K}^{0} N^{*}(1535) \rightarrow \bar{K}^{0} \eta p$ decay within the two approaches is different. For the $\Lambda_{c}^{+} \rightarrow \pi^{0} \phi p$ decay, we consider a triangle singularity mechanism, where the $\Lambda_{c}^{+}$decays into the $K^{*} \Sigma^{*}(1385)$, the $\Sigma^{*}(1385)$ decays into the $\pi^{0} \Sigma / \Lambda$, and then the $K^{*} \Sigma / \Lambda$ merge to produce the $\phi p$ in the final state. This mechanism produces a peak structure around $2020 \mathrm{MeV}$. In addition, the possibility that there is a hidden-strange pentaquark-like state is also considered by taking into account the final state interactions of $K^{*} \Lambda, K^{*} \Sigma$, and $\phi p$. We conclude that it is difficult to search for the hidden-strange state in this decay. However, we do expect nontrivial behavior in the $\phi p$ invariant mass distribution. The proposed $\Lambda_{c}^{+}$decay mechanism here can provide valuable information on the properties of these nuclear resonances and can in principle be tested by experiments such as BESIII, LHCb and Belle-II.
\end{abstract}

\section{Introduction}

The nonleptonic weak decays of charmed hadrons provide a useful platform to study hadronic resonances, some of which are subjects of intense debate about their nature [13]. For instance, the $\Lambda_{c}^{+} \rightarrow \pi^{+} M B$ weak decays are studied in Refs. $[4,5]$ from the viewpoint of probing the $\Lambda(1405)$ and $\Lambda(1670)$ resonances and extracting the $\pi \Sigma$ scattering lengths, where $M$ and $B$ stand for mesons and baryons. In Ref. [6], the $\Lambda(1670)$ and $a_{0}(980)$ resonances are investigated in the $\Lambda_{c}^{+} \rightarrow \pi^{+} \eta \Lambda$ decay. The role of the possible $\Sigma^{*}(1380)$ state with $J^{P}=1 / 2^{-}$in the $\Lambda_{c}^{+} \rightarrow \eta \pi^{+} \Lambda$ decay is also studied in Ref. [7] where the color-suppressed $W$ exchange diagram is considered for the production of the $\Sigma^{*}(1385)$ with $J^{P}=3 / 2^{+}$. Experimentally, in Ref. [8], the decays $\Lambda_{c}^{+} \rightarrow \eta \pi^{+} \Lambda$ and $\eta \Sigma^{*+}(1385)$ was investigated by the BESIII collaboration. On the other hand, in Ref. [9] the role of the exclusive $\Lambda_{c}^{+}$decays into a neutron in testing the flavor symmetry and final state interaction was proposed. It was shown that the three body nonleptonic decays of $\Lambda_{c}^{+}$ are of great interest to explore the final state interactions.

Analogous to the hidden-charm pentaquark states that were observed in the $J / \psi p$ invariant mass spectrum via the

*e-mail: xiejujun@impcas.ac.cn
$\Lambda_{b}^{0} \rightarrow K^{-} J / \psi p$ decay by the LHCb collaboration [10, 11], one may consider the possible existence of hidden-strange pentaquarks $P_{s}=u u d s \bar{s}$, in which the $c \bar{c}$ pair is replaced by $s \bar{s}$ [12]. In fact, in the light flavor sector below 2 $\mathrm{GeV}$, understanding the nature of the $N^{*}(1535)$ resonance with spin parity $J^{P}=1 / 2^{-}$is very challenging $[13,14]$. One peculiar property of the $N^{*}(1535)$ is that it couples strongly to the channels with strangeness, which is difficult to understand in the classical quark models. However, the strange decay properties of the $N^{*}(1535)$ resonance can be easily understood if it contains large five-quark components [15-18]. Within this pentaquark picture, the $N^{*}(1535)$ resonance could be the lowest $L=1$ orbitally excited $u u d$ state with a large admixture of $[u d][u s] \bar{s}$ pentaquark component. This makes the $N^{*}(1535)$ heavier than the $N^{*}(1440)$ and also gives a natural explanation of its large couplings to the channels with strangeness [19]. The large $N^{*}(1535)$ coupling to the $K \Lambda$ channel obtained in Refs. [15, 20] from the analysis of $J / \psi \rightarrow \bar{p} K^{+} \Lambda$ and $J / \psi \rightarrow \bar{p} p \eta$ reactions has been challenged in Ref. [21].

At around $2 \mathrm{GeV}$, a $\phi N$ bound state is predicted in several models [22-24]. Such a $\phi N$ states can be viewed as a $P_{s}$ pentaquark state. The forward-direction enhancement 
at around invariant mass $W=2.1 \mathrm{GeV}$ in the $\gamma p \rightarrow p \phi$ reaction $[25,26]$ may indicate the $s$-channel contributions from nucleon excited state around $2.1 \mathrm{GeV}$ [27].

In Ref. [28], the role of the $N^{*}(1535)$ resonance in the $\Lambda_{c}^{+} \rightarrow \bar{K}^{0} \eta p$ decay was investigated by taking the advantage of the strong coupling of the $N^{*}(1535)$ to the $\eta N$ channel and its large $u u d s \bar{s}$ component. While for the possible $P_{s}$ state, it was studied in Ref. [29] in the singly Cabibbo suppressed process $\Lambda_{c}^{+} \rightarrow \pi^{0} \phi p$, as shown in Fig. 1 in the quark level. However, the main difficulty is that the decay $\Lambda_{c}^{+} \rightarrow \pi^{0} \phi p$ has a very limited phase space [12].

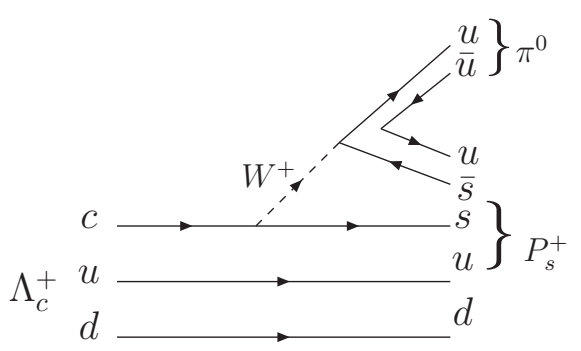

Figure 1. Quark level diagram for the $\Lambda_{c}^{+} \rightarrow \pi^{0} P_{s}^{+}$decay.

Furthermore, in Ref. [29], a contribution from triangle singularities close to the physical region was also considered to the $\Lambda_{c}^{+} \rightarrow \pi^{0} \phi p$ decay, where the $\Lambda_{c}^{+}$decays into $K^{*} \Sigma^{*}(1385)$, the $\Sigma^{*}(1385)\left(\equiv \Sigma^{*}\right)$ decays to the $\pi^{0} \Sigma$ (or $\Lambda$ ) and the $K^{*} \Sigma$ (or $\Lambda$ ) rescatter into $\phi p$ in the final state. In addition to the effects of the triangle mechanism, the final state interaction of $K^{*} \Lambda \rightarrow \phi p$ and $K^{*} \Sigma \rightarrow \phi p$ are also taken into account. If there was a $P_{s}$ resonance, it must couple to both the $\phi p$ and $K^{*} \Sigma / \Lambda$ and thus may be manifest in the $\phi p$ invariant mass distribution. Yet, because of the small phase space and depending on the mass and width of such a $P_{s}$ state, it could be difficult to search for it. Indeed, searching for the decay of $\Lambda_{c}^{+} \rightarrow \pi^{0} p \phi$ was done by the Belle Collaboration, and no significant signal was observed with an upper limit on the branching fraction of $\mathcal{B}\left(\Lambda_{c}^{+} \rightarrow \pi^{0} p \phi\right)<15.3 \times 10^{-5}$ at the $90 \%$ confidence level [30].

\section{The $\Lambda_{c}^{+} \rightarrow \bar{K}^{0} \eta p$ decay}

The quark content of $\Lambda_{c}^{+}$is $c u d$, where the up and down quarks are in a state of spin zero and isospin zero. Thus, these two light quarks are flavor antisymmetric, and the following simplified notation can be used for the $\Lambda_{c}^{+}$:

$$
\Lambda_{c}^{+}=\frac{1}{\sqrt{2}} \mid c(u d-d u)>
$$

In the $\Lambda_{c}^{+}$weak decay, the charm quark first decays into a strange quark by emitting a $W^{+}$boson, then the $W^{+}$decays into a pair of $\bar{d}$ and $u$ quarks, which is the most dominated process for the charmed baryon decays. In Fig. 2 we show the mechanism for the decay of $\Lambda_{c}^{+}$to produce the $\bar{K}^{0}$ from the $s \bar{d}$ pair and the insertion of a new $\bar{q} q$ pair with the quantum numbers of the vacuum, $\bar{u} u+\bar{d} d+\bar{s} s$, to construct the intermediate meson-baryon state $M B$ from the $u u d$ cluster with the assumption that the $u$ and $d$ quarks in the $\Lambda_{c}^{+}$are spectators in the weak decay. Thus, after the hadronization these $u$ and $d$ quarks in the $\Lambda_{c}^{+}$are part of the baryon, and the $u$ quark originated from the weak decay forms the meson. In this process, the $u d$ diquark in $\Lambda_{c}^{+}$is the spectator, and the $u u d$ cluster is combined into a pure $I=1 / 2$ state.

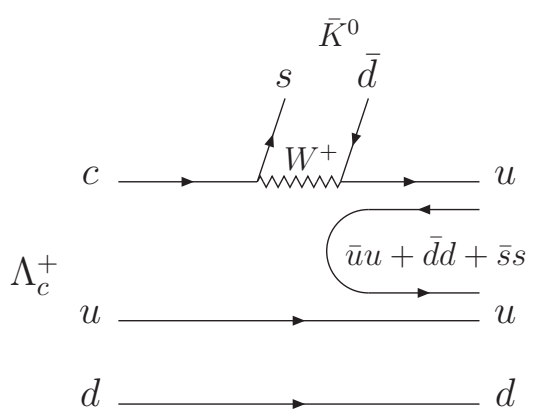

Figure 2. Quark level diagram for the $\Lambda_{c}^{+} \rightarrow \bar{K}^{0} M B$ decay with the $\bar{K}^{0}$ emission from the $s \bar{d}$ pair.

Following the procedure of Refs. [31-33], one can straightforwardly obtain the meson-baryon states after the $\bar{q} q$ pair production as

$$
|M B\rangle=\frac{\sqrt{3}}{3}|\eta p\rangle+\frac{\sqrt{2}}{2}\left|\pi^{0} p\right\rangle+\left|\pi^{+} n\right\rangle-\frac{\sqrt{6}}{3}\left|K^{+} \Lambda\right\rangle,
$$

where we have omitted the $\eta^{\prime} p$ term because of its large mass threshold compared to other channels that we considered.

After the production of a meson-baryon pair, the finalstate interaction between them takes place, which can be parameterized by the re-scattering shown in Fig. 3 at the hadronic level for the $\Lambda_{c}^{+} \rightarrow \bar{K}^{0} \eta p$ decay. Finally, the final-state interaction of $M B$, in $I=1 / 2$, will lead to the dynamical generation of the $N^{*}(1535)$ resonance $[34,35]$.
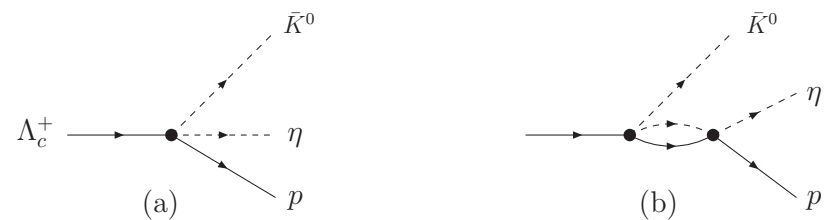

Figure 3. Diagrams for the $\Lambda_{c}^{+} \rightarrow \bar{K}^{0} \eta p$ decay: (a) direct $\bar{K}^{0} \eta p$ vertex at tree level, (b) final-state interaction of the $\eta p$.

According to Eq. (2), we can write down the $\Lambda_{c}^{+} \rightarrow$ $\bar{K}^{0} \eta p$ decay amplitude $T^{M B}$ of Fig. 3 as [36],

$$
\begin{aligned}
T^{M B}= & V_{P}\left(\frac{\sqrt{3}}{3}+\frac{\sqrt{3}}{3} G_{\eta p} t_{\eta p \rightarrow \eta p}+\frac{\sqrt{2}}{2} G_{\pi^{0} p} t_{\pi^{0} p \rightarrow \eta p}\right. \\
& \left.+G_{\pi^{+} n} t_{\pi^{+} n \rightarrow \eta p}-\frac{\sqrt{6}}{3} G_{K^{+} \Lambda} t_{K^{+} \Lambda \rightarrow \eta p}\right)
\end{aligned}
$$

where $V_{P}$ expresses the weak and hadronization strength, which is assumed to be a constant and independent of the 
final state interaction. The $G_{M B}$ denotes the one-mesonone-baryon loop function, which depends on the invariant mass of the final $\eta p$ system, $M_{\eta p}$. The meson-baryon scattering amplitudes $t_{M B \rightarrow \eta p}$ are those obtained in the chiral unitary approach, which depend also on $M_{\eta p}$.

On the other hand, because the $N^{*}(1535)$ has a large $u u d s \bar{s}$ component, it can also be produced via the process shown in Fig. 4 (a). After the $N^{*}(1535)$ is formed with $u u d s \bar{s}$, it decays into $\eta p$. The hadron level diagram for the decay of $\Lambda_{c}^{+} \rightarrow \bar{K}^{0} N^{*}(1535) \rightarrow \bar{K}^{0} \eta p$ is also shown in Fig. 4 (b). However, we should note that the strangeness component of $N^{*}(1535)$ can not be guaranteed from the decay process shown in Fig. 4. Indeed, the $N^{*}(1535)$ can also be produced from the process shown in Fig. 2, where the $s \bar{d}$ forms the $\bar{K}^{0}$, while the $N^{*}(1535)$ is constructed from the $u u d$ cluster and then it decays into $\eta p$ because of its large coupling to this channel.

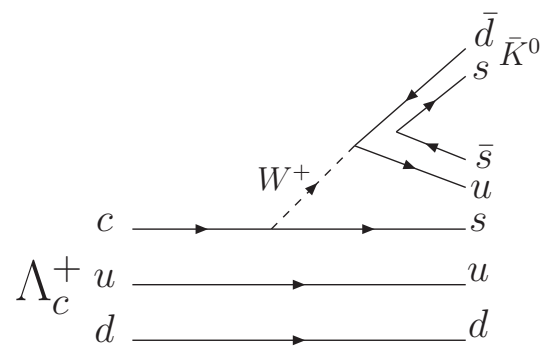

(a)

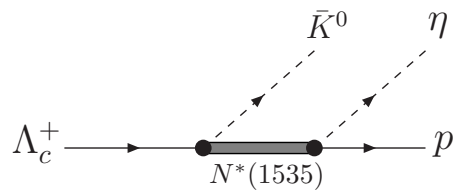

(b)

Figure 4. Quark level diagram for $\Lambda_{c}^{+} \rightarrow \bar{K}^{0} N^{*}$ (1535) (a) and hadron level diagram for $\Lambda_{c}^{+} \rightarrow \bar{K}^{0} \eta p$ decay (b).

The general decay amplitudes for $\Lambda_{c}^{+} \rightarrow \bar{K}^{0} N^{*}(1535)$, shown in Fig. 4, can be decomposed into two different structures with the corresponding coefficients $C_{1}$ and $C_{2}$,

$$
\mathcal{M}=i \bar{u}(q)\left(C_{1}+C_{2} \gamma_{5}\right) u(p),
$$

where $q$ and $p$ are the momentum of the $N^{*}(1535)$ and $\Lambda_{c}^{+}$, respectively. The coefficients $C_{1}$ and $C_{2}$ for charmed baryons decaying into ground meson and baryon states, in general, can be calculated in the framework of the pole model [37] or within the perturbative $Q C D$ approach [38]. In the present case, because the $N^{*}(1535)$ resonance is not well understood in the classical quark model, the values of $C_{1}$ and $C_{2}$ in Eq. (4) are very difficult to be pined down, and we have to determine them with future experimental data. In this work, we take $C_{1}=C_{2}$.

To get the whole decay amplitude of $\Lambda_{c}^{+} \rightarrow$ $\bar{K}^{0} N^{*}(1535) \rightarrow \bar{K}^{0} \eta p$ as shown in Fig. 4 (b), we use the effective interaction Lagrangian as in Ref. [39] for the
$N^{*}(1535) N \eta$ vertex,

$$
\mathcal{L}_{N^{*} N \eta}=-i g_{N^{*} N \eta} \bar{N} \eta N^{*}+\text { h.c.. }
$$

Then the invariant decay amplitude of the $\Lambda_{c}^{+} \rightarrow$ $\bar{K}^{0} N^{*}(1535) \rightarrow \bar{K}^{0} \eta p$ decay can be written as

$$
T^{N^{*}}=i g_{N^{*} N \eta} \bar{u}\left(p_{3}, s_{p}\right) G_{N^{*}}(q)\left(C_{1}+C_{2} \gamma_{5}\right) u\left(p, s_{\Lambda_{c}^{+}}\right),
$$

where $p_{3}$ is the momentum of the final proton. The $s_{p}$ and $s_{\Lambda_{c}^{+}}$are the spin polarization variables for the proton and $\Lambda_{c}^{+}$baryon, respectively. The $G_{N^{*}}(q)$ is the propagator of the $N^{*}(1535)$, which is given by a Breit-Wigner (BW) form as,

$$
G_{N^{*}}(q)=i \frac{\phi+M_{N^{*}}}{q^{2}-M_{N^{*}}^{2}+i M_{N^{*}} \Gamma_{N^{*}}\left(q^{2}\right)},
$$

with $M_{N^{*}}$ and $\Gamma_{N^{*}}\left(q^{2}\right)$ the mass and total decay width of the $N^{*}(1535)$, respectively. For $\Gamma_{N^{*}}\left(q^{2}\right)$, since the dominant decay channels for the $N^{*}(1535)$ resonance are $\pi N$ and $\eta N$ [40], we take the following form as used in Refs. [41, 42]

$$
\Gamma_{N^{*}}\left(q^{2}\right)=\Gamma_{N^{*} \rightarrow \pi N}\left(q^{2}\right)+\Gamma_{N^{*} \rightarrow \eta N}\left(q^{2}\right)+\Gamma_{0},
$$

with

$$
\begin{aligned}
& \Gamma_{N^{*} \rightarrow \pi N}\left(q^{2}\right)=\frac{3 g_{N^{*} N \pi}^{2} \frac{E_{1}+m_{p}}{4 \pi} \sqrt{E_{1}^{2}-m_{p}^{2}},}{\sqrt{q^{2}}} \\
& \Gamma_{N^{*} \rightarrow \eta N}\left(q^{2}\right)=\frac{g_{N^{*} N \eta}^{2}}{4 \pi} \frac{E_{2}+m_{p}}{\sqrt{q^{2}}} \sqrt{E_{2}^{2}-m_{p}^{2}} .
\end{aligned}
$$

Here

$$
E_{1}=\frac{q^{2}+m_{p}^{2}-m_{\pi}^{2}}{2 \sqrt{q^{2}}}, \quad E_{2}=\frac{q^{2}+m_{p}^{2}-m_{\eta}^{2}}{2 \sqrt{q^{2}}} .
$$

We take $g_{N^{*} N \pi}^{2} / 4 \pi=0.037$ and $g_{N^{*} N \eta}^{2} / 4 \pi=0.28$ as used in Ref. [43]. With these values we can get $\Gamma_{N^{*} \rightarrow N \pi}=67.5 \mathrm{MeV}$ and $\Gamma_{N^{*} \rightarrow N \eta}=63 \mathrm{MeV}$ if we take $\sqrt{q^{2}}=1535 \mathrm{MeV}$. In this work, we choose $\Gamma_{0}=19.5$ $\mathrm{MeV}$ for $\Gamma_{N^{*}}\left(\sqrt{q^{2}}=1535 \mathrm{MeV}\right)=150 \mathrm{MeV}$.

With all the ingredients obtained in the previous subsection, one can write down the invariant $\eta p$ mass distribution of the $\Lambda_{c}^{+} \rightarrow \bar{K}^{0} \eta p$ decay as:

$$
\frac{d \Gamma}{d M_{\eta p}}=\frac{1}{16 \pi^{3}} \frac{m_{p} p_{\bar{K}^{0}} p_{\eta}^{*}}{M_{\Lambda_{c}^{+}}}|T|^{2}
$$

where $T$ is the total decay amplitude. The $p_{\bar{K}^{0}}$ and $p_{\eta}^{*}$ are the three-momenta of the outgoing $\bar{K}^{0}$ meson in the $\Lambda_{c}^{+}$ rest frame and the outgoing $\eta$ meson in the center of mass frame of the final $\eta p$ system, respectively.

In Fig. 5, we show the numerical results for the $d \Gamma / d M_{\eta p}$ with two models: Model I takes $T=T^{M B}$, while Model II takes $T=T^{N^{*}}, M_{N^{*}}=1535 \mathrm{MeV}$ and $\Gamma_{N^{*}}$ is energy dependent as in Eq. (8). The results of Model I are obtained with $V_{P}=1 \mathrm{MeV}^{-1}$. The results of Model II are obtained with $C_{1}=C_{2}=47.2$, which are normalized to the peak of Model I.

For Model I, there is a clear peak around $1524 \mathrm{MeV}$ corresponding to the $N^{*}(1535)$ resonance. The peak position of Model II is very close to the central value, 1535 


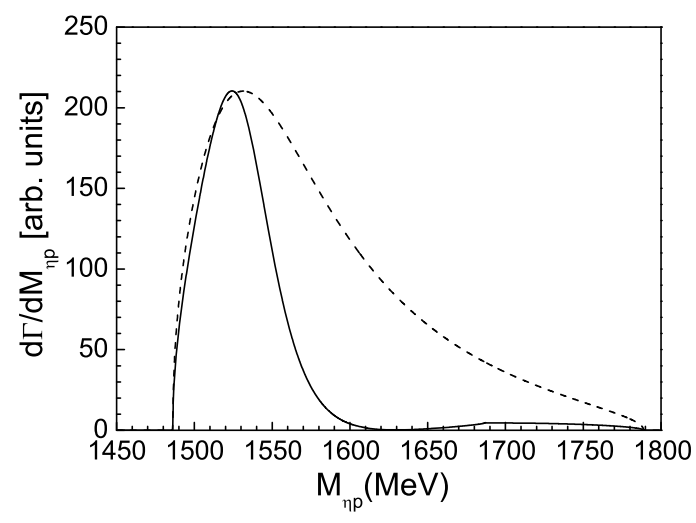

Figure 5. Invariant $\eta p$ mass distribution for the $\Lambda_{c}^{+} \rightarrow \bar{K}^{0} \eta p$ decay. The solid and dashed curves represent the results obtained in Model I and II, respectively.

$\mathrm{MeV}$, estimated in the PDG [40] for the $N^{*}(1535)$. The peak position of Model I is also close to the value 1535 $\mathrm{MeV}$, but with a narrow width. From the results of Model I and II shown in Fig. 5, we see that these two different descriptions of the $N^{*}(1535)$ resonance give different line shapes for the invariant $\eta p$ mass distributions. The findings here are similar to that obtained in Refs. [44, 45]. For the $N^{*}(1535)$, the amplitude square obtained with the chiral unitary approach does not behave like an usual BW resonance, even at the peak position. Note that in the chiral unitary approach, only the meson-baryon components of $N^{*}(1535)$ are included. However, in the works of Refs. [46, 47], it was shown that the $N^{*}(1535)$ contains a mixture of a genuine quark state apart from the mesonbaryon components.

Up to now, we have considered only the contribution from $N^{*}(1535)$, while the contributions from other baryon resonances, such as, $N^{*}(1650) \frac{1}{2}^{-}, N^{*}(1710) \frac{1}{2}^{+}$, $N^{*}(1720) \frac{3^{+}}{2}, \Sigma^{*}(1660) \frac{1}{2}^{+}, \Sigma^{*}(1670) \frac{3}{2}^{-}$, and $\Sigma^{*}(1750) \frac{1}{2}^{-}$, are not taken into account. In Fig. 6, we show the Dalitz plot for the $\Lambda_{c}^{+} \rightarrow \bar{K}^{0} \eta p$ decay, where we see that the the decay of $\Lambda_{c}^{+} \rightarrow \bar{K}^{0} N^{*}(1710)$ and $\Lambda_{c}^{+} \rightarrow \bar{K}^{0} N^{*}(1720)$ have very limited phase space, and $N^{*}(1710)$ and $N^{*}(1720)$ decay into $\eta p$ in $p$-wave, hence, their contributions should be much suppressed. On the other hand, in the $N^{*}(1535)$ energy region, the Dalitz plot overlaps with these $\Sigma^{*}$ resonances from 1600 to $1800 \mathrm{MeV}$ in the $\bar{K}^{0} p$ channel, which may make the analysis of $N^{*}(1535)$ difficult. Fortunately, the $\Sigma^{*}(1660) \frac{1^{+}}{2}$ and $\Sigma^{*}(1670) \frac{3}{2}^{-}$decay into $\bar{K}^{0} p$ in $p$-wave and $D$-wave, respectively. These contributions will be suppressed because of the higher partial waves involved. For the $\Sigma^{*}(1750) \frac{1^{-}}{2}$, it decays into $\bar{K}^{0} p$ in $s$-wave. However, it lies in the kinematic end-point region and therefore the decay of $\Lambda_{c}^{+} \rightarrow \eta \Sigma^{*}(1750)$ has a relative small phase space.

It is worthy to mention that both the $N^{*}(1535)$ and $N^{*}(1650)$ are dynamically generated from the analysis of the $s$-wave $\pi N$ scattering [48-50]. Indeed, the effect of the $N^{*}(1535)$ and the $N^{*}(1650)$ resonances are studied in Ref. [51], where both effects of the $N^{*}(1535)$ and the

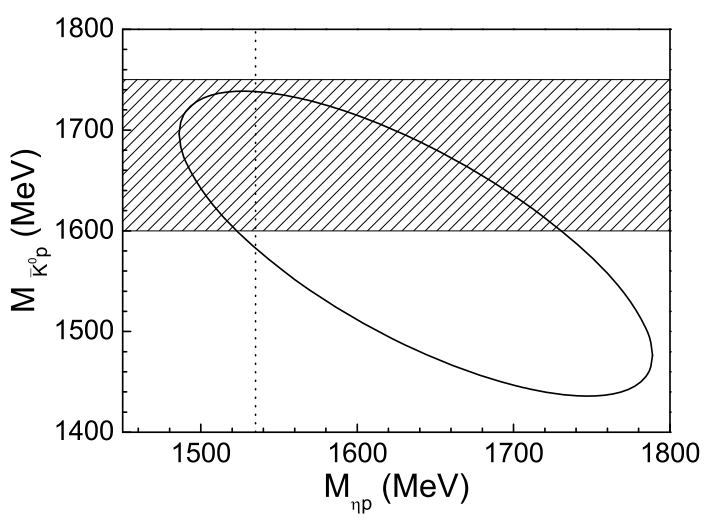

Figure 6. Dalitz plot for $M_{\eta p}$ and $M_{\bar{K}^{0} p}$ in the $\Lambda_{c}^{+} \rightarrow \bar{K}^{0} \eta p$ decay. The $N^{*}(1535)$ energy is shown by the vertical dotted line, which the horizontal band represents the masses of $\Sigma^{*}$ states from 1600 to $1800 \mathrm{MeV}$.

$N^{*}(1650)$ are seen in the mass distributions, but, their manifestation is different. Thus, the contributions from other $N^{*}$ and $\Sigma^{*}$ resonances might be small compared with the contribution from the $N^{*}(1535)$, and we expect that their contributions will not change much the model predictions here. If future experimental measurements provide enough data to disentangle the contributions from these resonances, one can also study them.

\section{The $\Lambda_{c}^{+} \rightarrow \pi^{0} \phi p$ decay}

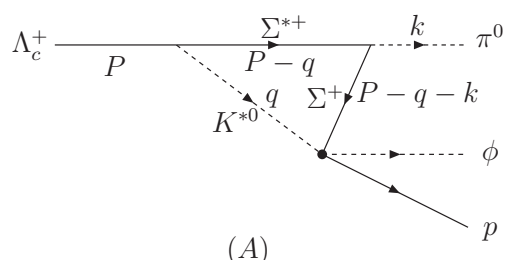

$(A)$

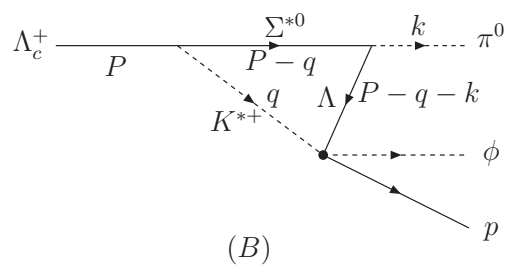

Figure 7. Triangle diagrams for the $\Lambda_{c}^{+} \rightarrow \pi^{0} p \phi$ decay. $(A): \Sigma^{+}-$ exchange. $(B): \Lambda$-exchange. The definitions of the kinematical variables $(P, q, k)$ are also shown.

In addition to the diagram shown in Fig. 1 , the $\Lambda_{c}^{+} \rightarrow$ $\pi^{0} p \phi$ decay can proceed through the triangle diagrams depicted in Fig. 7, where the decay of $\Lambda_{c}^{+} \rightarrow\left(\Sigma^{*}(1385) K^{*}\right)^{+}$ can proceed by $W$-exchange diagram [52, 53]: $(c d) u \rightarrow$ $(d u) u$, and the $d u u$ are hadronized, together with a $s \bar{s}$ 
pair with the vacuum quantum numbers, into the $\Sigma^{*}(1385)$ ( $\left.\equiv \Sigma^{*}\right)$ and $K^{*}$. Then the $\Sigma^{*}(1385)$ decays into $\pi^{0} \Sigma / \Lambda$ and, after the production of the $K^{* 0} \Sigma^{+}$and $K^{*+} \Lambda$, they re-scatter into the $\phi p$ in the final state, as shown in Fig. 7.

In Fig. 8, we show explicitly the $W$-exchange diagram at quark level for the decays $\Lambda_{c}^{+} \rightarrow \Sigma^{* 0}(1385) K^{*+}$ and $\Lambda_{c}^{+} \rightarrow \Sigma^{*+}(1385) K^{* 0}$.

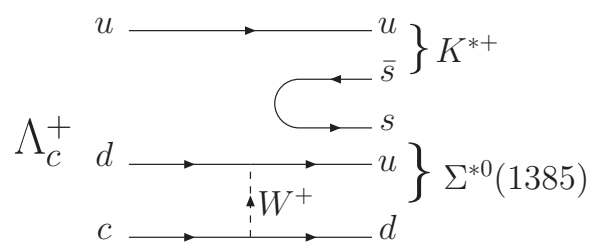

$(a)$

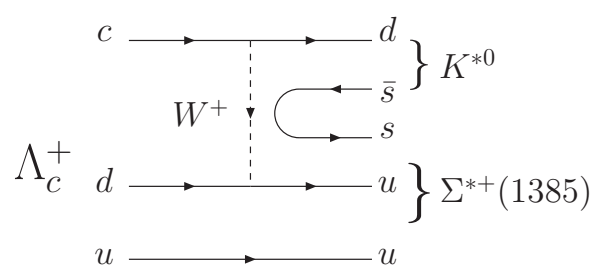

(b)

Figure 8. Quark level $W$-exchange diagram for the $\Lambda_{c}^{+} \rightarrow$ $\left(\Sigma^{*}(1385) K^{*}\right)^{+}$decay. $(a): \Lambda_{c}^{+} \rightarrow \Sigma^{* 0}(1385) K^{*+} .(B): \Lambda_{c}^{+} \rightarrow$ $\Sigma^{*+}(1385) K^{* 0}$.

Next, to get the total decay amplitude of the decay $\Lambda_{c}^{+} \rightarrow \pi^{0} \phi p$. We first need the effective interaction for the $\Lambda_{c}^{+} \rightarrow\left(\Sigma^{*} K^{*}\right)^{+}$vertex. Because the $\Sigma^{*} K^{*}$ threshold is very close to the mass of $\Lambda_{c}^{+}$, we consider only the $S$-wave coupling, which can be written as

$$
t_{\Lambda_{c}^{+} \rightarrow \Sigma^{*} K^{*}}=f_{I} g_{\Lambda_{c} \Sigma^{*} K^{*}} \bar{u}^{\mu}(P-q) u(P) \varepsilon_{\mu}(q),
$$

where $f_{I}$ is the isospin factor with $f_{I}=\sqrt{6} / 3$ for the $\Sigma^{*+} K^{* 0}$ and $-\sqrt{3} / 3$ for the $\Sigma^{* 0} K^{*+}$, and $g_{\Lambda_{c} \Sigma^{*} K^{*}}$ is an effective coupling constant which can be estimated, in general, from the branching faction of $\operatorname{Br}\left[\Lambda_{c}^{+} \rightarrow \Sigma^{*}(1385) K^{*}\right]$.

Second, we consider the $\Sigma^{*} \pi \Sigma$ and $\Sigma^{*} \pi \Lambda$ vertexes. Since he decays of $\Sigma^{*} \rightarrow \pi \Sigma$ and $\pi \Lambda$ are in $P$ waves, we can write

$$
\begin{aligned}
t_{\Sigma^{*+} \rightarrow \pi^{0} \Sigma^{+}} & =\frac{g}{m_{\pi}} \bar{u}(P-q-k) u^{\mu}(P-q) k_{\mu}, \\
t_{\Sigma^{* 0} \rightarrow \pi^{0} \Lambda} & =\sqrt{3} \frac{g}{m_{\pi}} \bar{u}(P-q-k) u^{\mu}(P-q) k_{\mu},
\end{aligned}
$$

with $g=0.69$ obtained from the total decay width $\Gamma_{\Sigma^{*}}=$ $37.13 \mathrm{MeV}$ and the branching fraction of $\operatorname{Br}\left(\Sigma^{*} \rightarrow \pi \Sigma\right)=$ $11.7 \%$ [40].
Then the total decay amplitude for the processes shown in Fig. 7 can be written as

$$
\begin{aligned}
t= & \frac{g_{\Lambda_{c} \Sigma^{*} K^{*}} g}{m_{\pi}} \vec{\epsilon}_{\phi} \cdot \vec{k} \sum_{i=\Sigma, \Lambda} C_{i} \int \frac{\mathrm{d}^{4} q}{(2 \pi)^{4}} \times \\
& \frac{i 2 m_{\Sigma^{*}}}{(P-q)^{2}-m_{\Sigma^{*}}^{2}+i m_{\Sigma^{*}} \Gamma_{\Sigma^{*}}} \frac{i}{q^{2}-m_{K^{*}}^{2}+i m_{K^{*}} \Gamma_{K^{*}}} \\
& \frac{i 2 m_{i}}{(P-q-k)^{2}-m_{i}^{2}+i \epsilon},
\end{aligned}
$$

where we have defined $C_{\Sigma}=\frac{\sqrt{6}}{3} t_{K^{* 0} \Sigma^{+} \rightarrow \phi p}$ and $C_{\Lambda}=$ $-t_{K^{*+} \Lambda \rightarrow \phi p}$, and $t_{K^{* 0} \Sigma^{+} \rightarrow \phi p}$ and $t_{K^{*+} \Lambda \rightarrow \phi p}$ are $T$-matrix elements for the re-scattering processes. As discussed before, the $K^{*} \Sigma^{*}$ mass threshold is close to the mass of $\Lambda_{c}^{+}$and the range of the $\phi p$ invariant mass for the decay of interest is very small, which allows us to make nonrelativistic approximation for all the involved baryons and vector mesons. Therefore, we take into account only $S$ wave interaction for the re-scattering.

In Ref. [54], the interaction of $K^{*} \Sigma, K^{*} \Lambda$ and $\phi p$ in $S$ wave is studied in the framework of the local hidden gauge formalism using a coupled-channels unitary approach. In the Isospin $I=1 / 2$ sector, one pole around $1980 \mathrm{MeV}$ is found, which couples strongly to the channels of $K^{*} \Sigma$, $K^{*} \Lambda$, and $\phi N$. In Fig. 9 we show the results of $\left|t_{K^{*+} \Lambda \rightarrow \phi p}\right|^{2}$ (solid cure) and $\left|t_{K^{* 0} \Sigma^{+} \rightarrow \phi p}\right|^{2}$ (dashed curve) in the model of Ref. [54].

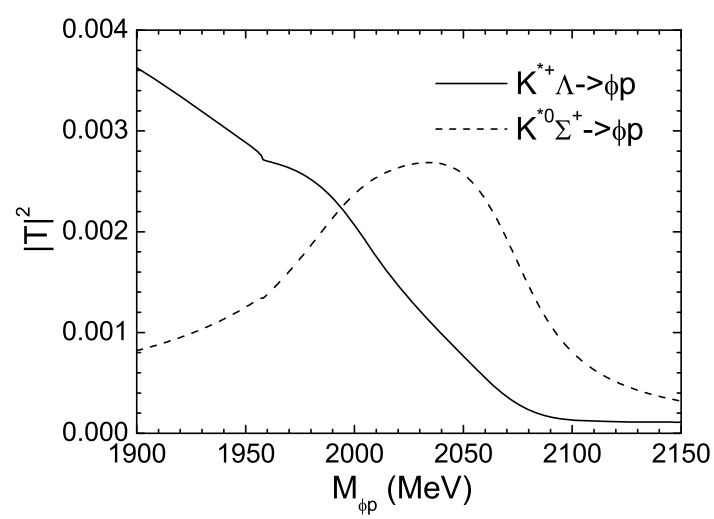

Figure 9. The square of the transition $T$-matrix elements for the $K^{*+} \Lambda \rightarrow \phi p$ and $K^{*+} \Sigma^{* 0} \rightarrow \phi p$ as a function of the $\phi p$ invariant mass $M_{\phi p}$ in the model of Ref. [54].

After performing the contour integration over the temporal component $q^{0}$ in Eq. (17), in the same way as shown in Refs. [55, 56], and including the finite widths of the $\Sigma^{*}$ and $K^{*}$ resonances, we get

$$
t=-\frac{g_{\Lambda_{c} \Sigma^{*} K^{*} g m_{\Sigma^{*}}}}{m_{\pi}} \vec{\epsilon}_{\phi} \cdot \vec{k} t_{T},
$$




$$
\begin{aligned}
t_{T}= & \sum_{i=\Sigma, \Lambda} C_{i} m_{i} \int \frac{\mathrm{d}^{3} q}{(2 \pi)^{3}} \frac{1}{\omega_{K^{*}} E_{\Sigma^{*}} E_{i}} \times \\
& \frac{1}{k^{0}-E_{i}-E_{\Sigma^{*}}+i \Gamma_{\Sigma^{*}} / 2} \frac{1}{P^{0}+\omega_{K^{*}}+E_{i}-k^{0}} \\
& \frac{1}{P^{0}-\omega_{K^{*}}-E_{i}-k^{0}+i \Gamma_{K^{*}} / 2} \\
& \frac{\left[P^{0} \omega_{K^{*}}+k^{0} E_{i}-\left(\omega_{K^{*}+E_{i}}\right)\left(\omega_{K^{*}}+E_{i}+E_{\Sigma^{*}}\right)\right]}{P^{0}-E_{\Sigma^{*}}-\omega_{K^{*}}+i \Gamma_{\Sigma^{*}} / 2}, \\
= & \frac{\sqrt{6} m_{\Sigma^{+}}}{3} t_{K^{* 0} \Sigma^{+} \rightarrow \phi p} t_{T}^{\Sigma}-m_{\Lambda} t_{K^{*+} \Lambda \rightarrow \phi p} t_{T}^{\Lambda},
\end{aligned}
$$

where $P^{0}=M_{\Lambda_{c}^{+}}$, and

$$
\begin{aligned}
& \omega_{K^{*}}=\sqrt{m_{K^{*}}^{2}+|\vec{q}|^{2}}, \quad E_{\Sigma^{*}}=\sqrt{m_{\Sigma^{*}}^{2}+|\vec{q}|^{2}}, \\
& k^{0}=\sqrt{m_{\pi^{0}}^{2}+|\vec{k}|^{2}}=\frac{M_{\Lambda_{c}^{+}}^{2}+m_{\pi^{0}}^{2}-M_{\phi p}^{2}}{2 M_{\Lambda_{c}^{+}}}, \\
& E_{i}=\sqrt{m_{i}^{2}+|\vec{q}+\vec{k}|^{2}} \text { with } i=\Sigma \text { or } \Lambda .
\end{aligned}
$$

With the masses of the initial state $\Lambda_{c}^{+}$, the neutral pion in the final state and two of the intermediate states, for example the $K^{*}$ and $\Lambda$, it is found that the region for the $\Sigma^{*}(1385)$ mass will produce a triangle singularity at the physical boundary $[57,58]$. However, because the $S$-wave vertices attached to the $\Lambda_{c}$ initial state and the $\phi p$ final state do not introduce any momentum dependence into the loop amplitude, and the $P$-wave pionic vertices results in a factor of the pion momentum, the above loop integral is ultraviolet convergent.

In Fig. 10 we plot the $\left|t_{T}^{\Lambda}\right|^{2}$ of the triangle loop defined in Eq. (18) for the $\Lambda$-exchange. It can be observed that $\left|t_{T}^{\Lambda}\right|^{2}$ has a peak around $2045 \mathrm{MeV}$, which is due to the triangle singularity [29].

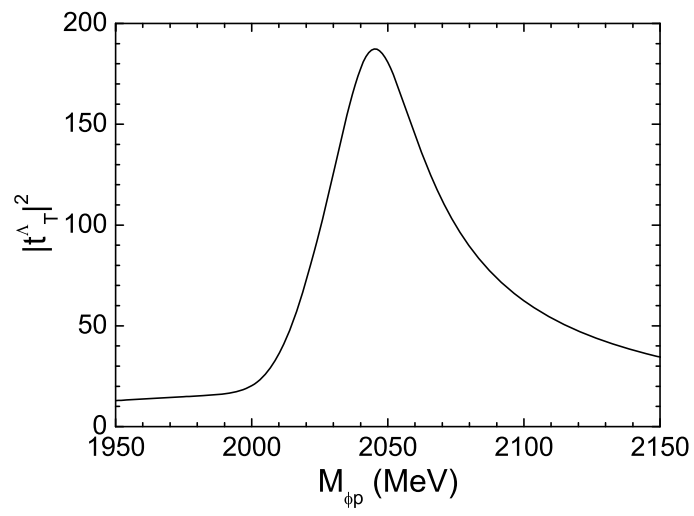

Figure 10. The square of the triangle amplitude $t_{T}^{\Lambda}$ of the process in Fig. 7(B) for the $\Lambda$-exchange as a function of the invariant mass of $\phi p$.

The $\phi p$ invariant mass mass distribution for the $\Lambda_{c}^{+} \rightarrow$ $\pi^{0} \phi p$ decay then reads [40]

$$
\frac{d \Gamma}{d M_{\phi p}}=\frac{m_{p} m_{\Sigma^{*}}^{2} g_{\Lambda_{c} \Sigma^{*} K^{*}}^{2} g^{2}}{16 \pi^{3} M_{\Lambda_{c}^{+}} m_{\pi}^{2}}|\vec{k}|^{3}\left|\vec{p}_{\phi}\right|\left|t_{T}\right|^{2},
$$

where $\vec{k}$ is the $\pi^{0}$ momentum in the rest frame of the $\Lambda_{c}^{+}$, and $\vec{p}_{\phi}$ is the $\phi$ momentum in the center-of-mass frame of the $\phi p$ system. Furthermore, we take also the approximation

$$
\sum\left|\vec{\epsilon}_{\phi} \cdot \vec{k}\right|^{2} \approx|k|^{2}
$$

where the sum runs over the polarization of $\phi$ meson.

Here we present the numerical results for the $\phi p$ invariant mass distribution, $\frac{d \Gamma}{d M_{\phi p}}$, for the $\Lambda_{c}^{+} \rightarrow \pi^{0} \phi p$ decay with three models: Model I represents the calculation of the triangle diagrams in Fig. 7 with the re-scattering $T$-matrix elements $t_{K^{*} \Sigma^{+} \rightarrow \phi p}$ and $t_{K^{*+} \Lambda \rightarrow \phi p}$ taken from Ref. [54], which includes the contribution of a $P_{s}$ state with a mass of about $1980 \mathrm{MeV}$; Model II is different from Model I by taking the $t_{K^{* 0} \Sigma^{+} \rightarrow \phi p}$ and $t_{K^{*+} \Lambda \rightarrow \phi p}$ as constants, and it thus represents the case without any $P_{S}$ resonance; Model III represents the results obtained from the pure phase space without any special dynamic, which we take the total decay amplitude $t_{\mathrm{III}}=c_{2}$. For the Model II, because of the very small phase space and the closeness of the mass thresholds, all of the involved hadrons, $K^{*}, \Sigma / \Lambda, \phi$ and proton, can be treated non-relativistically. Hence, one may construct a non-relativistic effective field theory describing the interaction between vector mesons and baryons with the leading order defined by a few constant contact terms, then the total decay amplitude $t_{\mathrm{II}}$ can be written as

$$
\begin{aligned}
t_{\mathrm{II}} & =t, \text { but with } t_{K^{*+} \Lambda \rightarrow \phi p}=\frac{\sqrt{6}}{2} t_{K^{* 0} \Sigma^{+} \rightarrow \phi p} \\
& =c_{1} \frac{\sqrt{6}}{2} \frac{E_{K^{*}}+E_{\phi}}{4 f_{\pi}^{2}},
\end{aligned}
$$

where $t$ is the amplitude shown in Eq. (17), and $c_{1}$ is constant. Besides, $E_{K^{*}}$ and $E_{\phi}$ are the energies of $K^{*+}$ and $\phi$ meson in the $\phi p$ rest frame. In this work we take $E_{K^{*}}=892 \mathrm{MeV}$ and $E_{\phi}=1043 \mathrm{MeV}$, which are obtained with the mass threshold of $K^{*+} \Lambda$. It is worth to mention that $t_{K^{*+} \Lambda \rightarrow \phi p}$ and $t_{K^{* 0} \Sigma^{+} \rightarrow \phi p}$ are calculated in the extreme non-relativistic limit from the vector mesons, while the method should works well in the present case which focuses on the immediate vicinity of the thresholds. There have been discussions about the validity of such an approximation in the case of vector-vector meson scatterings in the far-below threshold region, see, e.g., Refs. [59-62].

In Fig. 11, the solid, dashed, and dotted curves represent the results of Model I, II, and III, respectively. The parameter $c_{1}$ of Model II has been adjusted to the strength of the experimental data reported by the Belle Collaboration [30] at its peak around $M_{\phi p}=2020 \mathrm{MeV}$. The results of Model I and III are normalized such as to have the same integrated partial width as Model II. The result of Model II clearly shows a peak structure around $2.02 \mathrm{GeV}$. The origin of this peak is the triangle diagrams, in particular the $\Lambda$-exchange in Fig. 7 (B) which has a triangle singularity close to the $K^{*} \Lambda$ threshold $(\simeq 2.01 \mathrm{GeV})$. The width of this peak is comparable with the width of the $K^{*}$, which is about $50 \mathrm{MeV}$. This is a quite natural consequence as the $\phi p$ invariant mass is the same as the $K^{*} \Lambda$ invariant mass so that its distribution inherits the width of the $K^{*}$. The presence of a $P_{s}$ state with a mass close to the lower end 


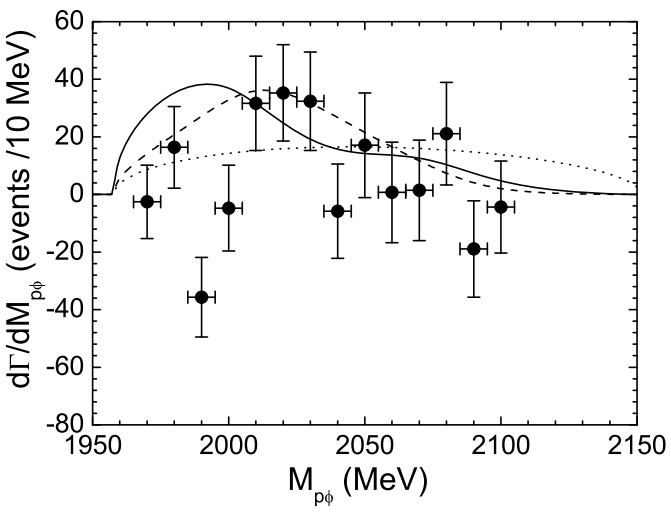

Figure 11. Invariant $\phi p$ mass distribution of the $\Lambda_{c}^{+} \rightarrow \pi^{0} p \phi$ decay. The experimental data are taken from Ref. [30].

of the $M_{\phi p}$ distribution results in a near-threshold enhancement. Identifying such an enhancement in experiments is difficult as it requires the data to have a high statistics. In particular, it becomes much more difficult if the $P_{s}$ mass is close to the $K^{*} \Lambda$ threshold because of the presence of kinematic singularities there. In any case, the phase space shown as Model III is very different from both Model I and Model II. Despite the low statistics of the current Belle data, the curve of Model II, whose shape is completely fixed, has a remarkable agreement with the data. In particular, the data seem to indeed show a bump structure around the $K^{*} \Lambda$ threshold. More data are welcome to clarify the situation.

\section{Summary}

We have studied the role of $N^{*}(1535)$ and possible $\phi p$ state in the decays of $\Lambda_{c}^{+} \rightarrow \bar{K}^{0} N^{*}(1535) \rightarrow \bar{K}^{0} \eta p$ and $\Lambda_{c}^{+} \rightarrow$ $\pi^{0} \phi p$, respectively. For the $\Lambda_{c}^{+} \rightarrow \bar{K}^{0} \eta p$ decay, we first employed the molecular picture where the $N^{*}(1535)$ is dynamically generated from the meson-baryon interaction. In such a scenario, the weak interaction part is dominated by the charm quark decay process: $c(u d) \rightarrow(s+u+\bar{d})(u d)$, while the hadronization part takes place by the $u u d$ cluster picking up a $q \bar{q}$ pair from the vacuum and hadronizes into a meson-baryon pair, while the $s \bar{d}$ pair from the weak decay turns into a $\bar{K}^{0}$. The following final state interactions of the meson-baryon pairs are described in the chiral unitary model that dynamically generates the $N^{*}(1535)$ resonance in the $I=1 / 2$ sector. Second, we take a Breit-Wigner formula to describe the distribution of the $N^{*}(1535)$ in the effective Lagrangian model. The above two descriptions for the $N^{*}(1535)$ resonance give different invariant $\eta p$ mass distributions. Future experimental measurements of the invariant $\eta p$ mass distribution studied in the present work will be very helpful to test our model calculations and constrain the properties of the $N^{*}(1535)$ resonance. For example, a corresponding experimental measurement could in principle be done at BESIII [63] and Belle.

For the $\Lambda_{c}^{+} \rightarrow \pi^{0} \phi p$ decay, which was proposed to be a channel to search for the hidden-strange $\phi p$ states, we take a mechanism that the $\Lambda_{c}^{+}$decays into the $K^{*}$ and $\Sigma^{*}(1385)$, then the $\Sigma^{*}(1385)$ subsequently decays into the $\left.\pi^{0} \Sigma / \Lambda\right)$, and the $K^{*}$ then interacts with the $\Sigma / \Lambda$ ) to produce the $\phi p$ in the final state. In the re-scattering of $K^{*} \Sigma / \Lambda \rightarrow \phi p$, we consider cases with and without a $\phi p$ state. For the case with the $\phi p$ state, we take $t_{K^{* 0} \Sigma^{+} \rightarrow \phi p}$ and $t_{K^{*+} \Lambda \rightarrow \phi p}$ as used in Refs. [54] which produces a resonance at around $1980 \mathrm{MeV}$. While for the case without the $\phi p$ state, both $t_{K^{* 0} \Sigma^{+} \rightarrow \phi p}$ and $t_{K^{*+} \Lambda \rightarrow \phi p}$ are constant. It is shown that the triangle singularities can produce a peak at around $2.02 \mathrm{GeV}$ with a width similar to that of the $K^{*}$. The theoretical results of the $\phi p$ invariant mass distribution agrees with the existing Belle data [30]. If there is a $P_{s}$ state, it could distort the $\phi p$ invariant mass distribution. However, it is difficult to be identified in the decay $\Lambda_{c}^{+} \rightarrow \pi^{0} \phi p$ because of its small phase space and the presence of triangle singularities. We look forward to more precise data from the BESIII, Belle-II and LHCb experiments in the future, which will be decisive to illuminate the role played by triangle singularities in this decay.

\section{Acknowledgments}

This work is partly supported by the National Natural Science Foundation of China under Grant Nos. 11735003, 11975041, 11747601, 11835015, 11961141012, 11475227, and the Youth Innovation Promotion Association CAS (2016367). It is also supported in part by the National Natural Science Foundation of China (NSFC) and the Deutsche Forschungsgemeinschaft (DFG) through the funds provided to the Sino-German Collaborative Research Center "Symmetries and the Emergence of Structure in QCD" (NSFC Grant No. 11621131001, DFG Grant No. TRR110), by the Chinese Academy of Sciences (CAS) under Grants No. QYZDB-SSW-SYS013 and XDPB09, and by the CAS Center for Excellence in Particle Physics (CCEPP).

\section{References}

[1] V. Crede and C. A. Meyer, Prog. Part. Nucl. Phys. 63, 74 (2009).

[2] H. X. Chen, W. Chen, X. Liu and S. L. Zhu, Phys. Rept. 639, 1 (2016).

[3] F. K. Guo, C. Hanhart, U. G. Meißner, Q. Wang, Q. Zhao and B. S. Zou, Rev. Mod. Phys. 90, 015004 (2018).

[4] K. Miyahara, T. Hyodo and E. Oset, Phys. Rev. C 92, 055204 (2015).

[5] T. Hyodo and M. Oka, Phys. Rev. C 84, 035201 (2011).

[6] J. J. Xie and L. S. Geng, Eur. Phys. J. C 76, 496 (2016).

[7] J. J. Xie and L. S. Geng, Phys. Rev. D 95, 074024 (2017).

[8] M. Ablikim et al. [BESIII Collaboration], Phys. Rev. D 99, 032010 (2019).

[9] C. D. Lü, W. Wang and F. S. Yu, Phys. Rev. D 93, 056008 (2016). 
[10] R. Aaij et al. [LHCb Collaboration], Phys. Rev. Lett. 115, 072001 (2015).

[11] R. Aaij et al. [LHCb Collaboration], Phys. Rev. Lett. 122, 222001 (2019).

[12] R. F. Lebed, Phys. Rev. D 92, 114030 (2015).

[13] E. Klempt and A. Zaitsev, Phys. Rept. 454, 1 (2007).

[14] V. Crede and W. Roberts, Rept. Prog. Phys. 76, 076301 (2013).

[15] B. C. Liu and B. S. Zou, Phys. Rev. Lett. 96, 042002 (2006).

[16] C. Helminen and D. O. Riska, Nucl. Phys. A 699, 624 (2002).

[17] B. S. Zou, Eur. Phys. J. A 35, 325 (2008).

[18] C. S. An and B. S. Zou, Eur. Phys. J. A 39, 195 (2009).

[19] B. S. Zou, Nucl. Phys. A 835, 199 (2010).

[20] B. C. Liu and B. S. Zou, Phys. Rev. Lett. 98, 039102 (2007).

[21] A. Sibirtsev, J. Haidenbauer and U.-G. Meissner, Phys. Rev. Lett. 98, 039101 (2007).

[22] H. Gao, H. Huang, T. Liu, J. Ping, F. Wang and Z. Zhao, Phys. Rev. C 95, 055202 (2017).

[23] H. Gao, T. S. H. Lee and V. Marinov, Phys. Rev. C 63, 022201 (2001).

[24] F. Huang, Z.-Y. Zhang and Y.-W. Yu, Phys. Rev. C 73, 025207 (2006).

[25] T. Mibe et al. [LEPS Collaboration], Phys. Rev. Lett. 95, 182001 (2005).

[26] K. Mizutani et al. [LEPS Collaboration], Phys. Rev. C 96, 062201 (2017).

[27] A. Kiswandhi, J. J. Xie and S. N. Yang, Phys. Lett. B 691, 214 (2010).

[28] J. J. Xie and L. S. Geng, Phys. Rev. D 96, 054009 (2017).

[29] J. J. Xie and F. K. Guo, Phys. Lett. B 774, 108 (2017).

[30] B. Pal et al. [Belle Collaboration], Phys. Rev. D 96, 051102 (2017).

[31] K. Miyahara, T. Hyodo, M. Oka, J. Nieves and E. Oset, Phys. Rev. C 95, 035212 (2017).

[32] L. Roca, M. Mai, E. Oset and U. G. Meißner, Eur. Phys. J. C 75, 218 (2015).

[33] A. Feijoo, V. K. Magas, A. Ramos and E. Oset, Phys. Rev. D 92, 076015 (2015).

[34] T. Inoue, E. Oset and M. J. Vicente Vacas, Phys. Rev. C 65, 035204 (2002).

[35] M. Doring, E. Oset and D. Strottman, Phys. Rev. C 73, 045209 (2006).

[36] J. A. Oller and E. Oset, Nucl. Phys. A 629, 739 (1998).

[37] H. Y. Cheng and B. Tseng, Phys. Rev. D 46, 1042 (1992) Erratum: [Phys. Rev. D 55, 1697 (1997)].
[38] C. D. Lü, Y. M. Wang, H. Zou, A. Ali and G. Kramer, Phys. Rev. D 80, 034011 (2009).

[39] J. J. Xie, B. S. Zou and H. C. Chiang, Phys. Rev. C 77, 015206 (2008).

[40] M. Tanabashi et al. [Particle Data Group], Phys. Rev. D 98, 030001 (2018).

[41] J. J. Wu, S. Dulat and B. S. Zou, Phys. Rev. C 81, 045210 (2010).

[42] J. J. Xie, B. C. Liu and C. S. An, Phys. Rev. C 88, 015203 (2013).

[43] Q. F. Lü, R. Wang, J. J. Xie, X. R. Chen and D. M. Li, Phys. Rev. C 91, 035204 (2015).

[44] L. S. Geng, E. Oset, B. S. Zou and M. Döring, Phys. Rev. C 79, 025203 (2009).

[45] M. Döring, E. Oset and B. S. Zou, Phys. Rev. C 78, 025207 (2008).

[46] D. Jido, M. Doering and E. Oset, Phys. Rev. C 77, 065207 (2008).

[47] T. Hyodo, D. Jido and A. Hosaka, Phys. Rev. C 78, 025203 (2008).

[48] E. J. Garzon and E. Oset, Phys. Rev. C 91, 025201 (2015).

[49] J. Nieves and E. Ruiz Arriola, Phys. Rev. D 64, 116008 (2001).

[50] P. C. Bruns, M. Mai and U. G. Meissner, Phys. Lett. B 697, 254 (2011).

[51] R. Pavao, S. Sakai and E. Oset, Phys. Rev. C 98, 015201 (2018).

[52] L.-L. Chau, H.-Y. Cheng and B. Tseng, Phys. Rev. D 54, 2132 (1996).

[53] H.-Y. Cheng and C.-W. Chiang, Phys. Rev. D 81, 074031 (2010).

[54] E. Oset and A. Ramos, Eur. Phys. J. A 44, 445 (2010).

[55] F. Aceti, J. M. Dias and E. Oset, Eur. Phys. J. A 51, 48 (2015).

[56] M. Bayar, F. Aceti, F.-K. Guo and E. Oset, Phys. Rev. D 94, 074039 (2016).

[57] F.-K. Guo, U.-G. Meißner, W. Wang and Z. Yang, Phys. Rev. D 92, 071502 (2015).

[58] X.-H. Liu, M. Oka and Q. Zhao, Phys. Lett. B 753, 297 (2016).

[59] D. Gülmez, U.-G. Meißner and J. A. Oller, Eur. Phys. J. C 77, 460 (2017).

[60] L. S. Geng, R. Molina and E. Oset, Chin. Phys. C 41, 124101 (2017).

[61] M. L. Du, D. Gülmez, F. K. Guo, U.-G. Meißner and Q. Wang, Eur. Phys. J. C 78, 988 (2018).

[62] R. Molina, L. S. Geng and E. Oset, PTEP 2019, 103B05 (2019).

[63] M. Ablikim et al. [BESIII Collaboration], Phys. Rev. Lett. 116, 052001 (2016). 